\title{
A Novel Approach to Reduce Signaling Delay in HMIPv6 mobile Networks
}

\author{
Sapna Gambhir, Member, IACSIT, M.N.Doja, Moinuddin and Mohit Gambhir
}

\begin{abstract}
- in the new era, changing requirements of users raise the need of next generation wireless networks (NGWN) which can provide efficient and quality of services to users any time any where. 4G wireless networks aim to provide best services, high data rates along with the permission to switch over across heterogeneous networks. In order to reduce signalling and registration delays in basic (MIPv6) networks, a new architecture HMIPv6 is considered. HMIPv6 architecture deals with local registration and global registration depending on the mobility.HMIPv6 architecture divides the mobility into two parts: micro-mobility and macro-mobility. The performance of HMIPv6 networks are immensely effected by selection of MAP that in turn includes selection of various attributes like speed of $\mathrm{MN}$, pattern followed by $\mathrm{MN}$ and type of the mobility (micro-mobility and macro-mobility) used by MN. In this paper, these three attributes are considered collectively for the selection of next best MAP in order to optimize various quality of service $(\mathrm{QoS})$ parameters viz. handoff delays, location update cost, packet delivery cost and signaling cost.

Proposed scheme encourages service providers to kept track of MNs history and then depending on the history and speed of MN, next best MAP is selected in order to multicast the packets before mobile node is actually connected to the selected MAP. Optimization of QoS parameters will finally result in providing remarkable communication experience to mobile phone users.
\end{abstract}

Index Terms - Hierarchical mobile IP (HMIP), Macro-mobility handoff, Micro-mobility handoff, Mobility Anchor Point (MAP), Mobile node (MN), Point of Attachment (PoA), Quality of Service (QoS).

\section{INTRODUCTION}

Mobile IPv6 protocol, standardized in IETF (Johnson et al, 2004), facilitates the roaming of MNs in different wired or wireless networks while maintaining a permanent IP address. This protocol provides continuous connectivity to MNs using care of address (CoA) specific to the point of Attachment (PoA) when MN moves from one PoA to another in different subnet. This process of movement of MN from PoA to another comes under handoff procedure. After moving to new PoA, MN must update home agent (HA) and correspondent node $(\mathrm{CN})$ about its new location. Increase in the change of PoA causes more location update cost, delays in packet delivery, handoff latency and QoS degradation. Use of HMIPv6 scheme overcomes these problems by focusing

Manuscript received October 10, 2008.

Sapna Gambhir is with YMCA Institute of Engineering, Faridabad, India; M N Doja is with Deptt. of Computer Engg., Faculty of Engineering, Jamia Milia Islamia, Delhi, India

Monniuddinn is with the Dr B R Ambedkar National Institute of Technology, Jalandhar, India

Mohit Gambhir is with TecEsq intellectual Property Solutions, Delhi, India; on reducing handover latency caused by the location update procedure.

In HMIPv6 (Soliman et al, 2005), an entity called MAP (mobility anchor point) is introduced which keeps track of user's mobility within a network domain. MN assigns two addresses, regional care-of-address (RCoA) and on-link-care-of-address (LCoA). Movement detection of MN is observed by the new IPv6 subnet prefix by listening to router advertisements (RA). Listening to RA, stateless auto-configuration of new $\mathrm{CoA}$ and duplicate address detection (DAD) adds handoff latency. Instead of relying on RA, a mobile-assisted link pre trigger (when MN initiates for handoff process) could be used to optimize or eliminate this movement detection delay.

By having an appropriate MAP selection that covers most of the MN's mobility area, we can significantly reduce the binding updates (global binding update and local binding update) to the HA and further reduce the signaling cost and location update cost in HMIP. The global binding update is a procedure in which $\mathrm{MN}$ registers its RCoA with the $\mathrm{CNs}$ and HA. On the other hand, a local binding update occurs when MN changes its current address within a local MAP domain, it only needs to register the new address with the MAP. In the mobile networks, signaling cost is proportional to the distance between two network entities resulting in larger location cost of global binding update in comparison to location cost of local binding update.

Objective of this paper is to improve the process of selection of next MAP with minimum frequency of handover and binding updates to the HA and hence improving handoff delays in HMIPv6 architecture of wireless networks.

Organization of this paper is as follows: Section 2 discusses the related work in HMIPv6 domain; section 3 introduces the proposed architecture of HMIPv6 and algorithm for MAP selection. Implementation of proposed work is explained in section 4 , section 5 analyzes the experimental results and section 6 concludes the paper.

\section{RELATED WORK}

In recent years, many researchers proposed advancements in HMIPv6 basic architecture in order to overcome the associated deficiencies. Mousavi (Mousavi et al, 2006) proposed a scheme to find an appropriate anchor point for each individual $\mathrm{MN}$ regarding its mobility pattern and its long term requested services. This scheme is not able to reduce message transition and address-binding delay and also fails in case of high speed MNs where their fast movements results in increased handovers and thus incurs more location update cost.

Soliman (Soliman et al, 2005) suggested using a distance-based selection algorithm, which selects the furthest 
MAP in order to reduce frequency of inter-domain handovers. Chung (Chung et al, 2007) considered that furthest MAP selection is inappropriate since it can be a performance bottleneck as the site grows or the number of ARs or MNs increases and therefore suggested two dynamic MAP selection algorithms for HMIPv6 networks LV-MAP and DV-MAP. These selections are based on user's mobility. LV-MAP scheme distributes load over multiple MAPs for an overloaded HMIPv6 network. DV-MAP selects the furthest MAP supporting MN's velocity for less overloaded situations with the aim to reduce the frequency of micro mobility handover (Hahaebi, 2006). Bandai (Bandai et al, 2003)] considered that Multilevel HMIPv6 is prone to load imbalances and therefore advocates that load-balancing to lower MAPs is necessary. Further, to reduce the number of BUs, M-HMIPv6 with speed estimation is proposed by Kawano (Kawano et al, 2002), in this scheme, by measuring the BU interval to the previous MAP, the MH's speed is estimated, and fast MH is connected to higher MAP. Pack (Pack et al, 2007) considered that it is difficult to estimate the velocity of $\mathrm{MNs}$ and the estimation results are often inaccurate. Therefore, MNs may not always register with an appropriate MAP. Location management method using MN's mobile history as discussed by Takashi (Takashi et al, 2004) calculates the area-covered rate of each upper MAP from mobile nodes mobile history and selects the MAP that best manages the $\mathrm{MN}$ in accordance with its rate.

The majority of existing proposals assume complete randomness of MNs movement. However, MNs move according to certain repetitive patterns. Illustrative examples of repetitive patterns of a common person are regular route to workplace, sport activities, weekend trips, vacations, etc. Hence based on this pattern, the best future MAP along the traveling path for each period of time can be easily predicted. If history-based prediction pattern for MAP selection algorithm is used then it would provide fast and efficient response to MN's handover request and resource allocation. It can also help to improve packet delivery cost because of route optimization.

In order to reduce service disruption delay, a bicasting tunnel scheme between the new and old MAP can be employed. With this scheme the new AR will have a copy of the on-going packets when the mobile node is still within the old MAP. When MN moves to a new MAP, new AR starts forwarding the packets to $\mathrm{MN}$ during the registration operation. In this paper, we propose a MAP selection algorithm based on the speed and history pattern of MN that reduces the handoff latency and hence improving location update and packet delivery.

\section{PROPOSED SCHEME}

Though MAP brings advantage to the mobile IP architecture by dividing the mobility into two i.e. micro-mobility and macro- mobility which further reduces location update cost and provides efficiency to the process of packet delivery, but the problem of selecting best MAP during handoff process remains. The AR advertisement does not specify which MAP is best for MN though there are often more than one optional MAP in area which result in frequent handovers and many binding updates. Problem of choice of best MAP during handoff process depends on many parameters. We consider speed of a mobile device and its history based pattern used by the mobile device recently during handoff process for MAP selection. Speed reflects the probability of change of PoA and history based pattern is used to choose best MAP by observing the same situation handled by MN in past. Details of process of MAP selection are discussed in next section.

\section{A. Proposed Handoff Algorithm}

Fig. 1 shows the change in the basic architecture of HMIPv6 for the proposed handoff process. In this changed architecture, each MAP maintains a new table i.e. history pattern table along with RLC table that was also handled by basic architecture. One more table is handled by HA which is RL table. Table I gives structure of history pattern table which would contain seven days track of ARs to which MN is connected i.e. 24*7 hrs MN tracks. Number of change of ARs or MAPs in a day depends on speed of MN and its mobility pattern which vary for each MN.

Table II shows RL table structure which contains information about current RCoA of $\mathrm{MN}$ so that packets can be sent to that RoA, this table is maintained by HA. Table III explains the structure of RLC table which contains information about MN's current LCoA so that packets can be sent to that LCoA, this table is maintained at MAP.

For handoff process, some assumptions are made, details of which are listed below and corresponding handoff algorithm is shown in Algorithm 1.

1. For optimized movement detection, Mobile-assisted pre-triggers are considered. The trigger in the form of a request control message would be generated by the $\mathrm{MN}$ when the received signal strength from the connected AR goes beyond a threshold value.

TABLE I: STRUCTURE OF HISTORY PATTERN TABLE

\begin{tabular}{|l|l|}
\hline Mobile Node IP address & $\begin{array}{l}\text { List of ARs used during } \\
\text { mobility }\end{array}$ \\
\hline & \\
\hline
\end{tabular}

TABLE II: STRUCTURE OF RC TABLE

\begin{tabular}{|l|l|}
\hline Mobile IP & RCOA \\
\hline & \\
\hline
\end{tabular}

TABLE III: STRUCTURE OF RLC TABLE

\begin{tabular}{|l|l|}
\hline Mobile IP & RCOA \\
\hline & \\
\hline
\end{tabular}




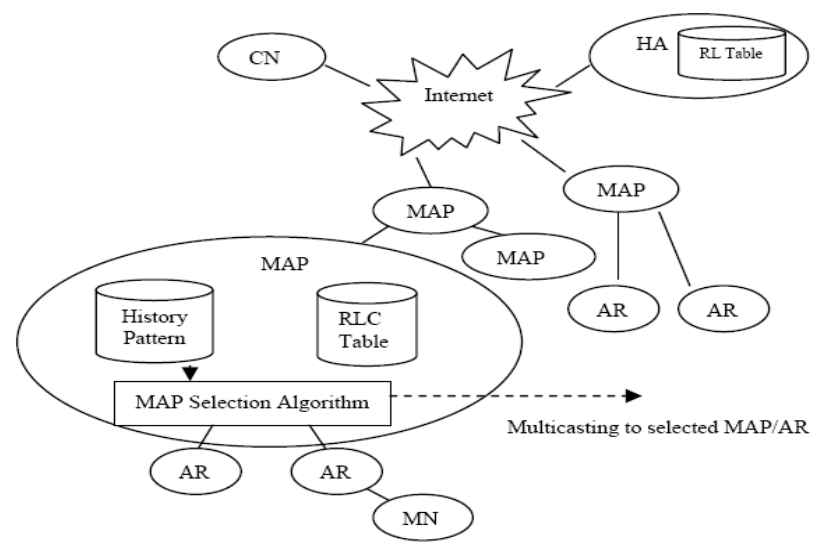

Fig.1 Proposed Architecture of HMIPv6

2. Multi-level hierarchy is assumed.

3. Highest MAP has complete topology information and is capable of communicating this information to lower MAPs and ARs.

4. Each MN keeps track of its last week history related to which MAPs /ARs served it their IP Addresses.

5. Constant average speed of Mobile Node is assumed.

For MAP selection process, speed of MN and history pattern used by MN, are two parameters for consideration. Speed of mobile device affects the probability of change of PoA. If a mobile device moves at very high speed then next MAP during handoff process depends on the data in history pattern table i.e. a MAP that is used in past for the similar situation would be used as a next MAP for micro-mobility handoff and multicasting packets to all AR during macro-mobility handoff process. If mobile device is moving with slow speed then next MAP selection depends on type of handoff. Next MAP is the parent of current MAP for the situation where $\mathrm{MN}$ is moving in the same MAP domain i.e. in case of macro mobility local location updating cost (cost for registrations to parent MAP for new PoA) is reduced. For the situation of micro-mobility handoff where parent MAP of old PoA and new PoA are different, next MAP is a MAP common to both domains and is at the least distance from both the domains which is a parent MAP of parent of old PoA and parent of new PoA.

Algorithm1: Handoff algorithm

Step 1: Initially, mobile node is connected to a particular access router within a MAP.

Step 2: As the MN starts the movement and the received signal strength from the AR goes beyond threshold signal strength, the Mobile node sends a request control message consisting of a request for next MAP selection, time at which request sent to the currently connected MAP and MN speed.

Step 3: After receiving the request, MAP initiates next MAP selection algorithm.

Step 4: Based on the results with of the MAP selection algorithm, MAP would create a bi-directional tunnel with the selected MAP and would forward the packets for MN to the new MAP.

$\mathrm{MN}$ with the new AR, the new MAP will forward packets to the MN

Algorithm 2: MAP Selection Algorithm
Step 1: Initialize threshold speed of mobile device.

Step 2: If the current speed of $\mathrm{MN}$ is lower than the threshold value and it migrates within same domain (intra-domain handoff) then the next AR within its own domain should be selected according to its history pattern stored in the database.

Step 3: If the speed of MN is lower than the threshold value of speed and it migrates within different MAP domain (inter-domain handoff) then next MAP should be selected according to its history pattern stored in the database.

Step 4: If the speed of the Mobile Node is more than the threshold speed and Mobile node is migrating fast within the same domain then multicast packets to all the access routers in the same domain.

Step 5: If the speed of the Mobile Node is more than the threshold speed and Mobile node is migrating fast between different domains then the next MAP would be the MAP common to both domains and is at the least distance from both of these domains.

\section{IMPLEMENTATION}

The algorithm is implemented using C\#, Macromedia-Flash 2008, SQL-2005 along with the basic version of HMIPv6. Various snapshots of the implementation are shown in Fig.2 (MN is connected in AR1), Fig.3 (result screen where AR2 forward the packets to the MN after the registration process as a result of execution of proposed algorithm). When the mobile node is about to leave the AR, it sends a pre-trigger to the MAP, informing it about the start of the handoff (Fig. 4). With the current speed and source AR, MAP calls proposed algorithm and then starts multicasting of packets to the newly selected AR or MAP (Fig 5). As the mobile node reaches the edge of the new AR, it sends a trigger to register itself with the new AR (Fig. 6). The MAP then stops multicasting and starts forwarding packets from the HA to the new AR. Similar process occurs when the MN changes the MAP domain. The change in the MAP domain is communicated to the HA. In case the MN's speed is very fast and it changes AR very frequently MAP multicasts the packets to all the ARs in its domain (Fig. 7). If the MAP crossing rate of the $\mathrm{MN}$ is high, then a higher-level MAP which is common to both domains and is at the least distance from both of these domains is selected (Fig. 8).

\section{EXPERIMENTAL RESULTS}

After implementation, proposed algorithm is compared with basic HMIPv6 functionality with respect to various QoS parameters like handoff delay, cell residency time and static and dynamic MNs. These parameters are discussed in this section.

\section{A. QoS Parameters}

In HMIPv6 architecture, handoff delay is considered as the time taken by $\mathrm{MN}$ to switch to new PoA which includes delay 
to listen router advertisements, delay for acknowledgements, one hop transmission delay in wired link, transmission delay in wireless link. Here, we consider handoff delay as time taken by MN to change its PoA and ready to continue with the acceptance of new packets. Thus the time taken to forward packets (which are collected by MAP during registration process) to $\mathrm{MN}$ through new PoA is considered under handoff delay resulting in delays which are mentioned above along with delay to transfer packets from MAP to AR and further AR to MN.

With this definition of handoff delay, basic HMIPv6 architecture includes all the above delays but proposed algorithm excludes the delay of transfer of packets from MAP to new PoA (i.e. AR) as the execution of the proposed algorithm and transfer of packets to new PoA is done in parallel to the registration process of $\mathrm{MN}$ to new PoA.

Further, we divide the MN movements in to two i.e. slow moving $\mathrm{MN}$ as static $\mathrm{MNs}$ and fast moving $\mathrm{MN}$ as dynamic MNs. With this, four situations are analyzed during handoff process. First situation explains slow moving $\mathrm{MN}$ macro-mobility handoff process; second situation says about slow moving MN micro-mobility handoff process, Third situation explains fast moving MN macro-mobility handoff process and last shows fast moving $\mathrm{MN}$ macro-mobility handoff process. These handoffs are calculated with some modifications of equations discussed by Lei (Lei et al, 2007) as shown below.

Macro-mobility and micro-mobility handoff delays (delays with the use of proposed scheme and delay with basic HMIPv6 functionality) are shown in Table $\mathrm{V}$ and corresponding graphs are shown in Fig. 9 and Fig. 10.With the graph, it is analyzed average percentage of delay of $35 \%$ is saved in using the proposed algorithm. Detail of saving of handoff delay in each situation is shown in Table VI and respective signaling diagrams are shown in Fig. 11 and Fig 12. Details of various parameters used in the signaling diagram are shown in Table IV.

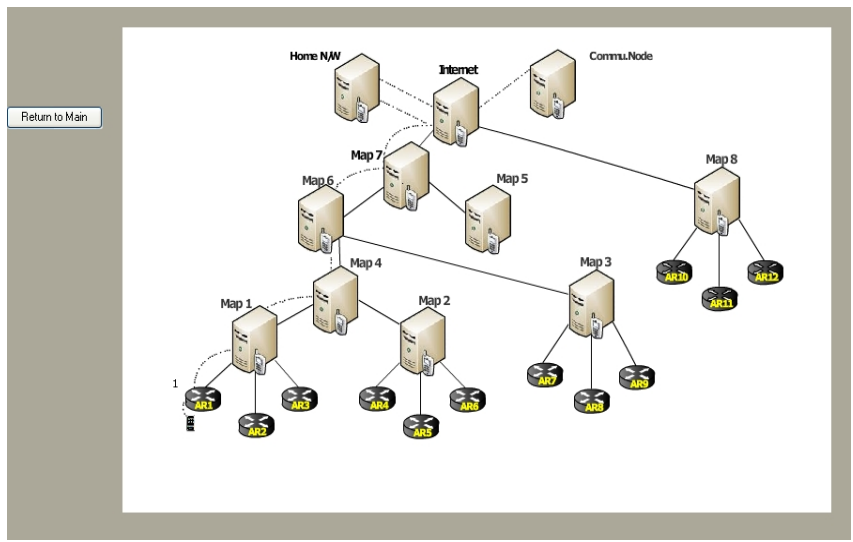

Fig 2: MN connected to AR1

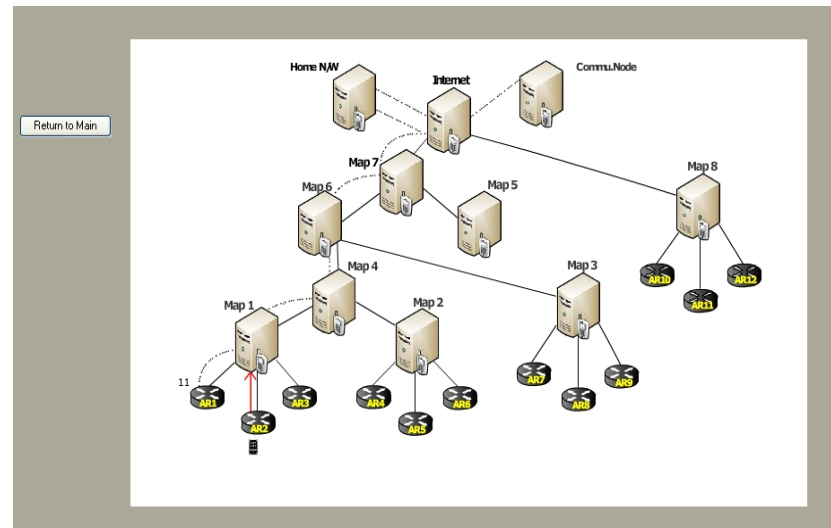

Fig 3: MN registers with AR2.and AR2 already have packets for $\mathrm{MN}$ as a result of proposed algorithm.

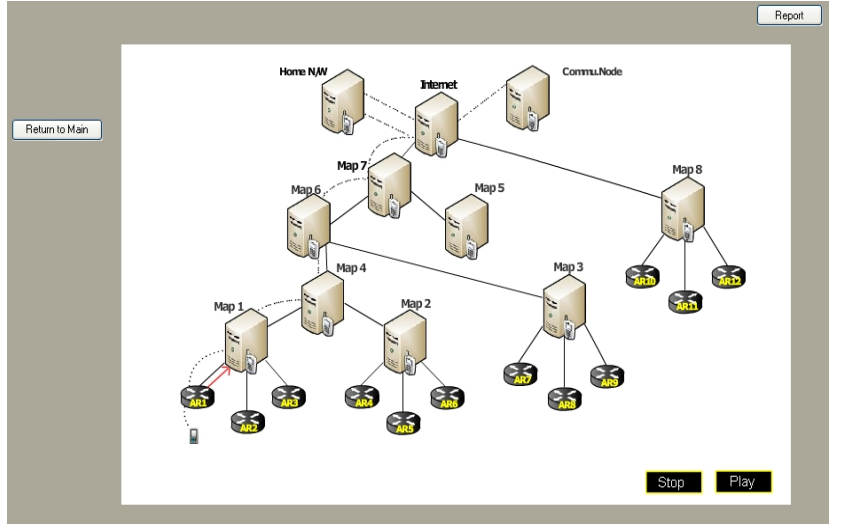

Fig 4: MN sending pre-trigger to MAP

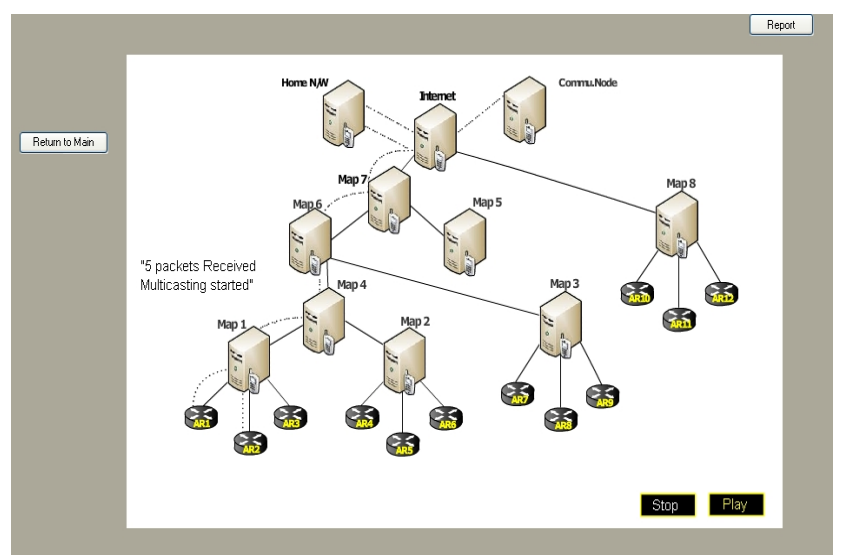

Fig 5: MAP multicasts packet to AR2

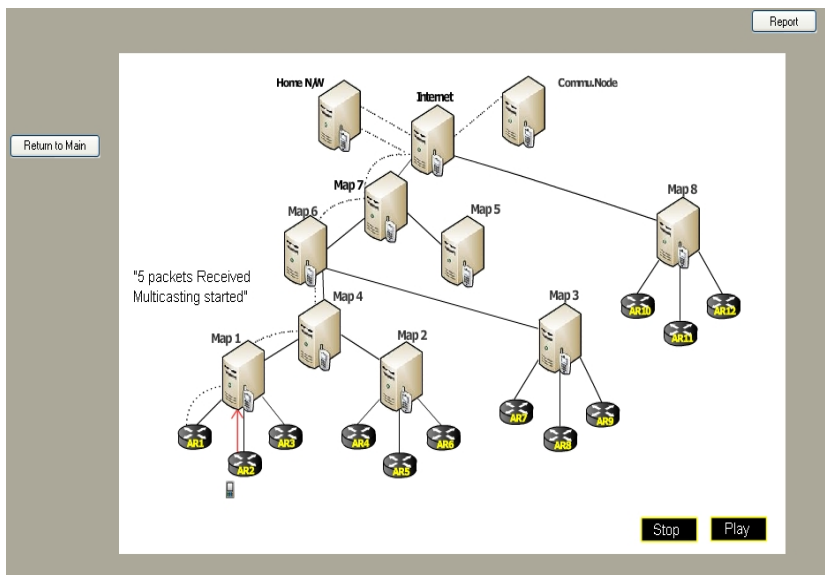

Fig 6: MN informs MAP of new ARF 


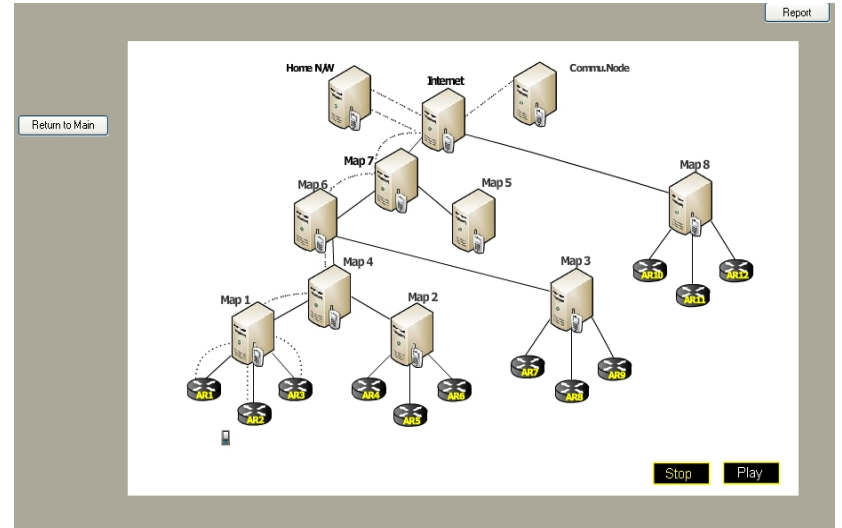

Fig 7: Multicasting to all in AR's in MAP1 domain

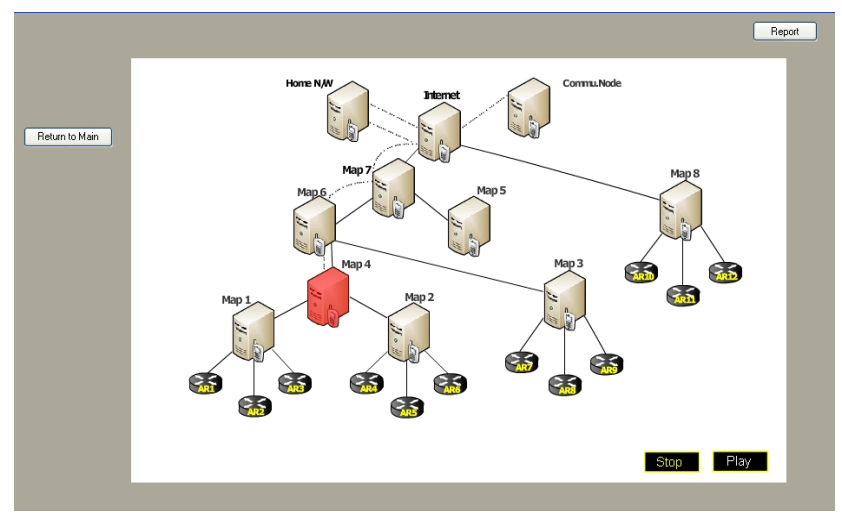

Fig 8: MN migrates frequently between MAP1 and MAP2, thus, MAP4 selected as parent MAP

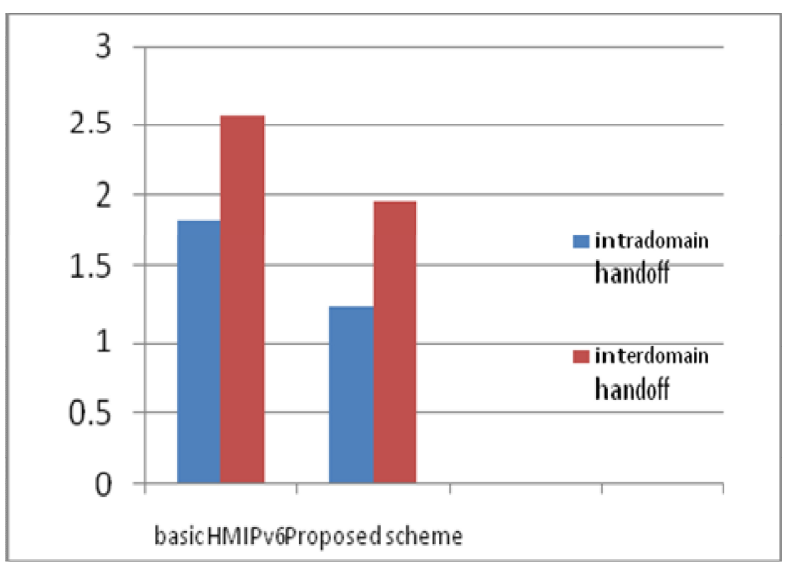

Fig 9: Handoff delay of basic version \& proposed scheme for Static MN

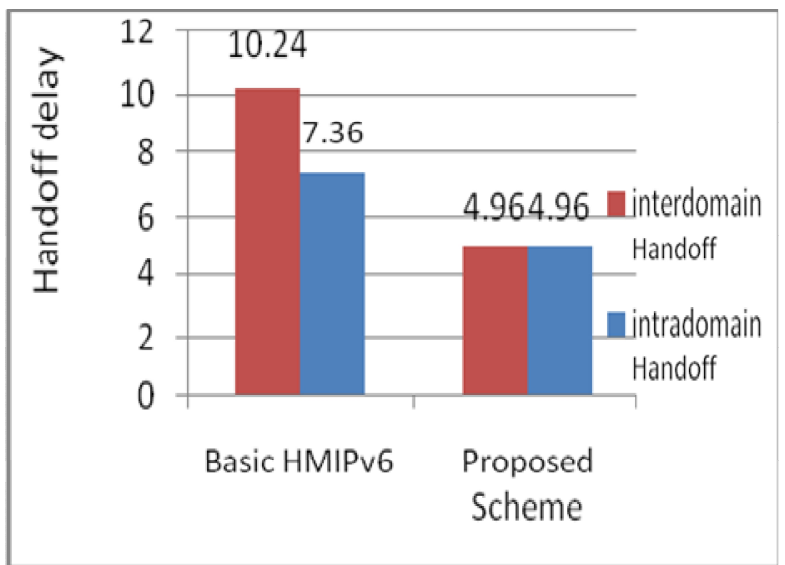

Fig. 10: Handoff delay of basic version and proposed scheme for dynamic $\mathrm{MN}$

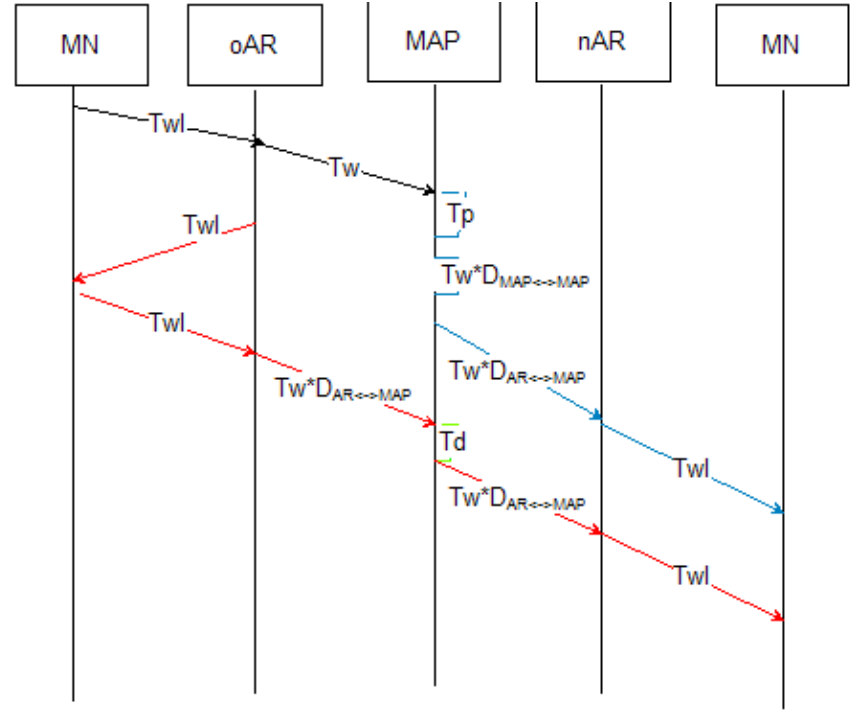

Fig 11: Micro mobility handoff signaling Diagram

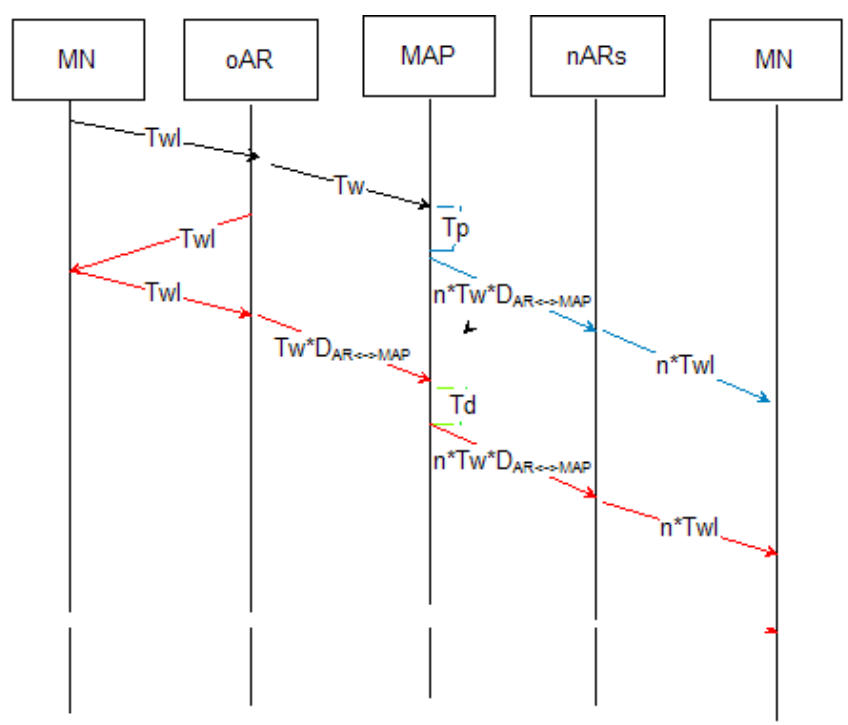

Fig 12: Macro mobility handoff signaling Diagram

\section{CONCLUSION}

In HMIPv6 architecture, selection of higher level MAP causes more chances for occurrence of macro-mobility handoff than micro-mobility handoff that further reduces location update cost but selection of lower MAP causes reduction in the delay of packet delivery cost which is received by MAP during handoff process. In the proposed MAP selection algorithm, more parameters viz. history pattern and speed of the mobile device are used to select next best MAP for call continuation and further reducing the delays during handoff process at an average of $35 \%$, only if the MAP or AR as a result of the algorithm is same as the $\mathrm{MAP}$ registered by the $\mathrm{MN}$.

Proposed scheme would help mobile phone industry to provide best, efficient and remarkable services to the users in near future.A conclusion section is not required. Although a conclusion may review the main points of the paper, do not replicate the abstract as the conclusion. A conclusion might elaborate on the importance of the work or suggest applications and extensions. 
TABLE IV: VALUES OF SOME PARAMETERS FOR CONSIDERATION

\begin{tabular}{|c|c|}
\hline $\begin{array}{l}\mathrm{T}_{\text {wl }} \\
\end{array}$ & Delay in wired link \\
\hline $\mathrm{T}_{\mathrm{w}}$ & Transmission delay in wireless link \\
\hline $\mathrm{D}_{\mathrm{AR}} \leftrightarrow \mathrm{MAP}$ & Distance between AR and MAP \\
\hline $\mathrm{n}$ & No. of packets \\
\hline $\mathrm{D}_{\mathrm{MAP} \leftrightarrow \mathrm{MAP}}$ & $\begin{array}{l}\text { Distance between Source MAP and next } \\
\text { predicted MAP }\end{array}$ \\
\hline $\begin{array}{l}\mathrm{T} \\
\mathrm{p}\end{array}$ & Time to execute proposed algorithm \\
\hline $\mathrm{T}_{\mathrm{d}}$ & $\begin{array}{l}\text { Time to decision module to check } \\
\text { if(algo(result)==MN(result)) }\end{array}$ \\
\hline
\end{tabular}

\section{REFERENCES}

[1] Bandai, M., Sasase, I. (2003), A Load Balancing Mobility Management for Multilevel Hierarchical Mobile IPv6 Networks, IEEE International Symposium on Personal, Indoor and Mobile Communication, Vol. 1, pp.460 - 464.

[2] D. Johnson, C Perkins, and J Arkko (2004), Mobility Support in IPv6, IETF RFC3775.

[3] Hesham Soliman, Claude Catelluccia Karim El Malki, and Ludovic Bellier (2005), Hierarchical Mobile IPv6 Mobility Management, IETF RFC4140.

[4] Kawano, K., Kinoshita, K. and Murakami, K. (2002), A Multilevel hierarchical distributed IP mobility management scheme for wide area networks, IEEE 11th International conference on Computer communication and networks, pp.480 - 484.

[5] Kumagai Takashi, Asaka Takuya and Takahashi Tatsuro (2004), Location Management Using Mobile History for Hierarchical Mobile IPv6 Networks, IEICE Trans. Common.; vol. 3, pp. 1585 - 1589.

[6] M.H. Hahaebi (2006), Macro/micro-mobility Fast handover in hierarchical Mobile IPv6, Computer Communications, vol. 29, pp. 611-617.

[7] Mousavi, M and Quintero, A (2006), Selection Mechanism in Hierarchical Mobile IP, IEEE International Conference wireless and mobile computing, Networking and communications, pp. $321-328$.

[8] Pack Sangheon, Kwon Tackyoung and Choi Yanghee (2007), A Performance comparison of mobility anchor point selection schemes in Hierarchical Mobile IPv6 networks, Elsevier Computer Networks; vol. 51, no.6, pp. 1630-1642.

[9] WonSik Chung and SuKyoung Lee (2007), Cost-Effective MAP Selection in HMIPv6 Networks, IEEE International Conference on communications, pp. $6026-6031$.

[10] Yi-Xue-Lei and Geng-Sheng Kuo (2007), Impact of MAP selection on handover performance for multimedia services in multi-level HMIPv6 networks, IEEE WCNC 2007, pp 3901-3906.

TABLE V. MACRO AND MICRO-MOBILITY HANDOFF DELAYS

\begin{tabular}{|l|l|l|l|l|}
\hline & $\begin{array}{l}\text { Macro-mobility } \\
\text { handoff(Static } \\
\text { MN) }\end{array}$ & $\begin{array}{l}\text { Micro-mobility } \\
\text { handoff(Static } \\
\text { MN) }\end{array}$ & $\begin{array}{l}\text { Macro-mobility } \\
\text { handoff(Dynamic } \\
\text { MN) }\end{array}$ & $\begin{array}{l}\text { Micro-mobility } \\
\text { handoff(dynamic } \\
\text { MN) }\end{array}$ \\
\hline Basic HMIPv6 & 1.84 & 2.56 & 7.36 & 4.96 \\
\hline Proposed Scheme & 1.24 & 1.96 & 10.24 & 4.96 \\
\hline
\end{tabular}

TABLE VI. PERCENTAGE OF SAVING OF HANDOFF DELAY IN DIFFERENT SITUATIONS USING PROPOSED SCHEME

\begin{tabular}{|l|l|l|l|l}
\hline & $\begin{array}{l}\text { Micro-mobility } \\
\text { handoff(Static MN) }\end{array}$ & $\begin{array}{l}\text { Micro-mobility } \\
\text { handoff(dynamic MN) }\end{array}$ & $\begin{array}{l}\text { Macro-mobility } \\
\text { handoff(Static MN) }\end{array}$ & $\begin{array}{l}\text { Macro-mobility } \\
\text { handoff(Dynamic MN) }\end{array}$ \\
\hline$\%$ of saving of handoff delay & 23.44 & 51.56 & 32.61 \\
\hline
\end{tabular}

